\title{
Surface electronic structure of the $\mathrm{Fe}_{3} \mathrm{O}_{4}(100)$ : Evidence of a half-metal to metal transition
}

\author{
M. Fonin, ${ }^{1, *}$ R. Pentcheva, ${ }^{2}$ Yu. S. Dedkov, ${ }^{3}$ M. Sperlich, ${ }^{4}$ D. V. Vyalikh, ${ }^{3}$ M. Scheffler, ${ }^{5}$ U. Rüdiger, ${ }^{1}$ and G. Güntherodt ${ }^{4}$ \\ ${ }^{1}$ Fachbereich Physik, Universität Konstanz, 78457 Konstanz, Germany \\ ${ }^{2}$ Department of Earth and Environmental Sciences, University of Munich, Theresienstr. 41, 80333 Munich, Germany \\ ${ }^{3}$ Institut für Festkörperphysik, Technische Universität Dresden, 01062 Dresden, Germany \\ ${ }^{4}$ II. Physikalisches Institut, Rheinisch-Westfälische Technische Hochschule Aachen, 52056 Aachen, Germany \\ ${ }^{5}$ Fritz-Haber-Institut der Max-Planck-Gesellschaft, Faradayweg 4-6, 14195 Berlin, Germany
}

(Received 27 June 2005; published 29 September 2005)

\begin{abstract}
In situ prepared $\mathrm{Fe}_{3} \mathrm{O}_{4}(100)$ thin films were studied by means of scanning tunneling microscopy (STM) and spin-polarized photoelectron spectroscopy (SP-PES). The atomically resolved $(\sqrt{2} \times \sqrt{2}) R 45^{\circ}$ wavelike surface atomic structure observed by STM is explained based on density functional theory (DFT) and ab initio atomistic thermodynamics calculations as a laterally distorted surface layer containing octahedral iron and oxygen, referred to as a modified $B$ layer. The work-function value of the $\mathrm{Fe}_{3} \mathrm{O}_{4}(100)$ surface extracted from the cutoff of the photoelectron spectra is in good agreement with that predicted from DFT. On the $\mathrm{Fe}_{3} \mathrm{O}_{4}(100)$ surface both the SP-PES measurements and the DFT results show a strong reduction of the spin polarization at the Fermi level $\left(E_{F}\right)$ compared to the bulk density of states. The nature of the states in the majority band gap of the $\mathrm{Fe}_{3} \mathrm{O}_{4}$ surface layer is analyzed.
\end{abstract}

DOI: 10.1103/PhysRevB.72.104436

PACS number(s): 75.25.+z, 71.30. $+\mathrm{h}$, 68.35.Bs, 68.47.Gh

\section{INTRODUCTION}

Magnetic transition metal oxides have recently attracted renewed attention due to the unique properties, such as metal-insulator phase transitions, ${ }^{1,2}$ superconductivity, ${ }^{3}$ and colossal magnetoresistance. ${ }^{4-6}$ Among them magnetite $\left(\mathrm{Fe}_{3} \mathrm{O}_{4}\right)$, a ferrimagnet with $T_{C}=851 \mathrm{~K}$, is an important material owing to its technological applications in catalysis and magnetic recording media. Moreover, $\mathrm{Fe}_{3} \mathrm{O}_{4}$ has been theoretically predicted to be a half-metallic ferrimagnetic material with a conductive minority spin channel and semiconducting majority spin channel, ${ }^{7,8}$ which results in a $100 \%$ spin polarization at $E_{F}$. This makes magnetite a prospective material for spintronic devices. ${ }^{9-11}$

$\mathrm{Fe}_{3} \mathrm{O}_{4}$ crystallizes in the cubic inverse spinel structure (space group $F d 3 m, a=8.396 \AA$ ) where the oxygen anions $\left(\mathrm{O}^{2-}\right)$ form a close-packed face-centered-cubic (fcc) sublattice with $\mathrm{Fe}^{2+}$ and $\mathrm{Fe}^{3+}$ cations located in interstitial sites. ${ }^{1}$ Two different kinds of cation sites exist in the magnetite crystal: tetrahedrally coordinated $A$ sites occupied by $\mathrm{Fe}_{A}$ (typically assigned with a charge state $3+$ ), and octahedrally coordinated $B$ sites occupied by $\mathrm{Fe}_{B}$ (typically assigned with charge states $2+$ and $3+$ in equal numbers). The bulk crystallographic structure of magnetite can be represented as a stack of (100) layers containing either iron atoms in tetrahedral $A$ sites or oxygen and iron ions in octahedral $B$ sites [Fig. 1(a)]. In the $B$ layers, only half of the octahedral sites are occupied and $\mathrm{Fe}$ ions form close-packed rows along the [011] direction. In successive $B$ planes these rows are arranged perpendicular to each other.

The two different bulk truncations of $\mathrm{Fe}_{3} \mathrm{O}_{4}(100)$ either with an $A$ layer containing $\mathrm{Fe}_{A}$ ions or with a $B$ layer consisting of oxygen and $\mathrm{Fe}_{B}$ ions [see Fig. 1(b)] are considered to be polar according to the classification given by Tasker. ${ }^{12}$ The previous understanding was that such surfaces must reconstruct to minimize the electrostatic energy. A $(\sqrt{2}$ $\times \sqrt{2}) R 45^{\circ}$ reconstruction has been experimentally observed on the $\mathrm{Fe}_{3} \mathrm{O}_{4}(100)$ surface. ${ }^{13-21}$ During the last decade, several possible structural models were proposed for the $(\sqrt{2}$ $\times \sqrt{2}) R 45^{\circ}$ reconstruction ${ }^{13-15,17-22}$ but the atomic arrangement of the $\mathrm{Fe}_{3} \mathrm{O}_{4}(100)$ surface has been a matter of discussion.

Chambers et al. ${ }^{18}$ investigated the $\mathrm{Fe}_{3} \mathrm{O}_{4}(100)$ surface using x-ray photoelectron spectroscopy (XPS) and x-ray photoelectron diffraction (XPD). Applying the autocompensation rule ${ }^{23}$ the authors concluded that the surface structure is a half-filled $A$ layer with strong inward relaxations of the surface layer. More recently, low energy ion scattering (LEIS) experiments ${ }^{26}$ also favored this termination. In a molecular dynamics calculation with classical potentials Rustad et al. ${ }^{22}$ proposed a 0.5 ML $A$ termination where the surface and half of the subsurface $\mathrm{Fe}_{A}$ relax towards octahedral positions in the surface $B$ layer.

In contrast, Voogt et al. ${ }^{17}$ suggested a structure consisting of a $B$-terminated surface with oxygen vacancies. The possibility of ordered hydroxyl groups was also discussed in this

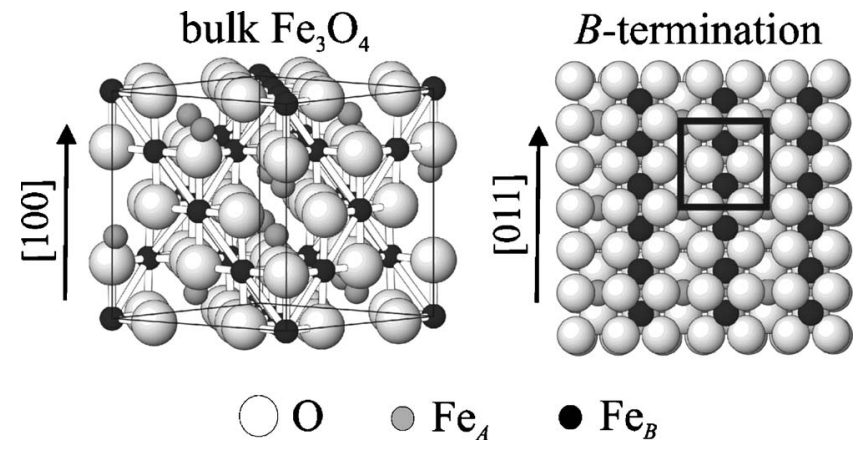

FIG. 1. (a) Side view of the inverse spinel $\mathrm{Fe}_{3} \mathrm{O}_{4}$ structure. (b) Top view of the $\mathrm{Fe}_{3} \mathrm{O}_{4}(100)$ bulk $B$-termination. The black open square in (b) marks the $p(1 \times 1)$ bulk unit cell. 
work. ${ }^{17}$ The former model of the $B$-terminated Fe surface with oxygen vacancies has been supported in a STM study by Stanka et al. ${ }^{19}$ The STM images show rows of octahedral iron atoms exhibiting a wavelike structure which was attributed to relaxations away from the missing oxygen atoms in the $B$ layer. ${ }^{19}$ Also, a noncompensated termination with charge ordered $B$ layer was suggested from STM measurements performed using ferro- ${ }^{27}$ or antiferromagnetic tips. ${ }^{20,21}$ However, the observed magnetic contrast alone cannot provide a clue on the stabilization mechanism of the surface. Bipolaronic effects in the $B$ termination have also been discussed as a possible interpretation of the magnetic contrast obtained with an antiferromagnetic STM tip. ${ }^{21}$

Recently, using $a b$ initio atomistic thermodynamics Pentcheva et $a .^{24}$ compiled a surface phase diagram for a variety of stoichiometric and nonstoichiometric configurations and found that the lowest energy configuration is a modified bulk termination with a $(\sqrt{2} \times \sqrt{2}) R 45^{\circ}$ periodicity which does not fulfill the autocompensation rule. ${ }^{24}$ The ideal $(1 \times 1)$ termination was found to be Jahn-Teller instable. The theoretical results are confirmed by qualitative $\mathrm{x}$-ray diffraction ${ }^{24}$ (XRD) and low-energy electron diffraction (LEED) analyses. ${ }^{25}$

The electronic band structure of $\mathrm{Fe}_{3} \mathrm{O}_{4}$ films has been extensively investigated by means of $\mathrm{x}$-ray magnetic circular dichroism, ${ }^{28,29}$ ultraviolet photoelectron spectroscopy, ${ }^{30-32}$ as well as spin- and angle-resolved photoelectron spectroscopy. ${ }^{33-37}$ Nevertheless, the interpretation of the valence-band photoemission spectra of $\mathrm{Fe}_{3} \mathrm{O}_{4}$ has been and still is a matter of debate. ${ }^{30,34-36}$ Recent investigations, ${ }^{30,36}$ however, showed that band dispersions ${ }^{7,8}$ must be taken into account for the interpretation of photoelecton spectra of $\mathrm{Fe}_{3} \mathrm{O}_{4}$.

Alvarado et al. performed the first spin-resolved photoelectron spectroscopy measurements on $\mathrm{Fe}_{3} \mathrm{O}_{4}(100)$ single crystals. ${ }^{33-35}$ The spin polarization $P(h \nu)$ of the photoelectrons with energies up to $11.2 \mathrm{eV}$ was measured by a Mott scattering detector. A maximum value of $-60 \%$ near the Fermi energy $\left(E_{F}\right)$ was measured at around $5 \mathrm{eV}$ photon energy at $10 \mathrm{~K} .{ }^{34}$ From a single-ion-in-a-crystal-field (SICF) model a maximum value of the spin polarization at $T=0 \mathrm{~K}$ is $P=-66.6 \%$ (Refs. 34 and 35) which was considered to be in agreement with the measured value of $-60 \%$.

Recently, spin polarization measured on the $\mathrm{Fe}_{3} \mathrm{O}_{4}(111)$ surface at $E_{F}$ at room temperature (RT) by means of spinpolarized angle-resolved photoemission spectroscopy (SPARPES) was reported to be as high as $-(80 \pm 5) \%$ which can be an evidence of the half-metallic nature of the magnetite in [111] direction. ${ }^{36}$ However, for the (100)-oriented $\mathrm{Fe}_{3} \mathrm{O}_{4}$ thin films the spin-resolved photoelectron spectroscopy measurements of thin epitaxial films grown on $\mathrm{MgO}(100)$ substrates yielded spin polarization values of only -(40-55)\% near $E_{F} \cdot{ }^{38,39}$ These values are significantly lower than the $-100 \%$ predicted by local spin density approximation (LSDA) calculations for the bulk material ${ }^{8}$ as well as the $-(80 \pm 5) \%$ measured on the (111)-oriented $\mathrm{Fe}_{3} \mathrm{O}_{4}$ surface at $E_{F}$ at RT. ${ }^{36}$ The low value for the (100) surface was ascribed either to surface imperfection ${ }^{38}$ or to strong electron correlation effects. ${ }^{39}$
In the present paper we report a combined experimental and theoretical study of the crystallographic as well as electronic surface structure of epitaxial $\mathrm{Fe}_{3} \mathrm{O}_{4}(100)$ thin films. Experimentally, in situ prepared, well-characterized $\mathrm{Fe}_{3} \mathrm{O}_{4}(100)$ films were studied by means of LEED, STM, and SP-PES. On the theoretical side, DFT calculations are performed to investigate the surface atomic arrangement as well as the electronic structure of $\mathrm{Fe}_{3} \mathrm{O}_{4}(100)$. The contrast in measured STM images is interpreted on the basis of STM simulations. The work-function value of the $\mathrm{Fe}_{3} \mathrm{O}_{4}(100)$ surface extracted from the cutoff of the photoelectron spectra is compared to the value predicted for the lowest energy configuration by the DFT calculations. Furthermore, the spin polarization at the Fermi energy is measured via SP-PES and compared to the value obtained from the DFT calculations. Finally, based on the DFT calculations, we analyze how the symmetry breaking at the surface as well as of the surface reconstruction alter the electronic properties.

\section{EXPERIMENTAL}

\section{A. Experimental setups}

The STM experiments were carried out in an ultra-high vacuum (UHV) system with a base pressure of 8 $\times 10^{-11}$ mbar equipped with LEED optics, a gas inlet, e-beam evaporation cells, and an Omicron UHV AFM/STM. All STM measurements were carried out at RT using electrochemically etched polycrystalline tungsten tips cleaned in UHV by $\mathrm{Ar}^{+}$sputtering. The presented STM images were taken in the constant-current mode.

The SP-PES measurements were carried out at U125/1PGM beamline at the BESSY II storage ring. The UHV system for SP-PES (base pressure $8 \times 10^{-11} \mathrm{mbar}$ ) consists of a UHV chamber equipped with LEED optics, a gas inlet, e-beam evaporation cells, and an Omicron SPLEED spin analyzer. The SP-PES spectra were recorded in normal emission. The total energy resolution and the acceptance angle were set to $100 \mathrm{meV}$ and $6^{\circ}$, respectively.

The work function as well as x-ray absorption spectroscopy measurements (XAS) were performed in an UHV system located at the Russian-German Laboratory at the BESSY II storage ring. An UHV chamber (base pressure 1 $\times 10^{-10}$ mbar) equipped with LEED optics, a gas inlet, e-beam evaporation cells, and a $127^{\circ}$ CLAM4 analyzer was used for these measurements. XAS spectra have been collected in the total electron yield mode and normalized to the maximum intensity.

\section{B. Sample preparation}

The epitaxial $\mathrm{Fe}_{3} \mathrm{O}_{4}(100)$ films used for STM and SP-PES measurements were prepared on $\mathrm{MgO}(100)$ single crystals. The epitaxial $\mathrm{Fe}_{3} \mathrm{O}_{4}(100)$ films for the work-function measurements were prepared on a $\mathrm{W}(100)$ single crystal. The $\mathrm{MgO}(100)$ substrates were cleaned in acetone, isopropanol, and methanol before the introduction into UHV and finally annealed in situ in $5 \times 10^{-6} \mathrm{mbar}_{2}$ for $2 \mathrm{~h}$ at about $600{ }^{\circ} \mathrm{C}$. The W(100) single crystal was cleaned in situ by using standard cleaning procedure: heating in $\mathrm{O}_{2}$ at $1800 \mathrm{~K}$ followed 


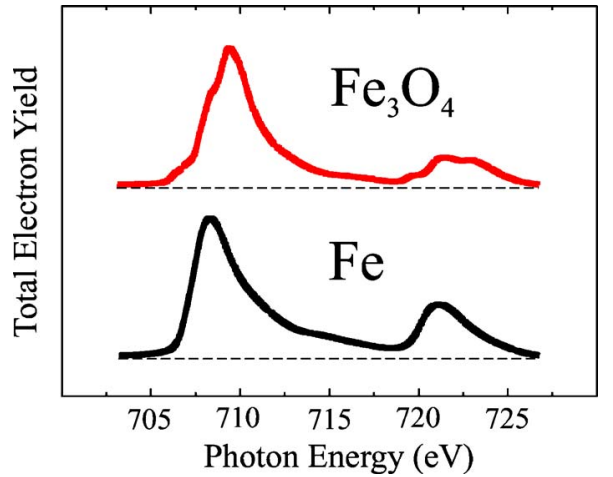

FIG. 2. (Color online) Fe $L_{2,3}$ edge XAS spectra of an epitaxial $\mathrm{Fe}_{3} \mathrm{O}_{4}(100)$ film (upper spectrum) as well as of the epitaxial $\mathrm{Fe}(100)$ film (lower spectrum).

by flashing up to $2300 \mathrm{~K}$. Fe was evaporated from high purity rods heated by electron bombardment. High quality 150-300 $\AA$ thick $\mathrm{Fe}_{3} \mathrm{O}_{4}(100)$ films were prepared in situ by Fe evaporation on $\mathrm{MgO}(100)$ or $\mathrm{W}(100)$ substrates in $\mathrm{O}_{2}$ atmosphere. During the growth, the $\mathrm{O}_{2}$ pressure was maintained at $2 \times 10^{-6}$ mbar and moderate substrate temperatures of about $300{ }^{\circ} \mathrm{C}$ were used to prevent magnesium interdiffusion in case of $\mathrm{MgO}(100)$ substrates. The high degree of crystalline order of the epitaxial $\mathrm{Fe}_{3} \mathrm{O}_{4}(100)$ films obtained in our experiments has been checked by LEED and STM. The surface cleanliness has been monitored by valence-band as well as core-level photoemission. Figure 2 presents the Fe $L_{2,3}$ edge XAS spectrum of the epitaxial $\mathrm{Fe}_{3} \mathrm{O}_{4}(100)$ film in comparison with that of the epitaxial $\mathrm{Fe}(100)$ film. The characteristic multiplet structure of the XAS spectrum at the $\mathrm{Fe}$ $L_{2,3}$ and the $\mathrm{O} K$ absorption edges (not shown here) of $\mathrm{Fe}_{3} \mathrm{O}_{4}$ agree with earlier experiments, ${ }^{28,29}$ confirming good bulk quality of the prepared samples.

\section{Theoretical methods}

The DFT calculations were performed using the fullpotential augmented plane waves (FP-APW) method in the WIEN2K implementation ${ }^{40}$ within the generalized gradient approximation $(\mathrm{GGA})^{41}$ of the exchange-correlation functional. The $\mathrm{Fe}_{3} \mathrm{O}_{4}(100)$ surface was modeled in the supercell geometry where the slab for, e.g., the $B$ termination, consists of seven $B$ layers and six $A$ layers. The vacuum between the repeated slabs amounts to $10 \AA$. The lateral parameter of the supercell is set to the GGA bulk lattice constant, $8.42 \AA$, which is in good agreement with the experimental value of $8.394 \AA$. The muffin tin (MT) radii of both chemical species are $R_{\mathrm{Fe}}^{\mathrm{MT}}=1.90$ bohr and $R_{\mathrm{O}}^{\mathrm{MT}}=1.40$ bohr. A mixed augmented plane wave $(\mathrm{APW}+\mathrm{lo})$ and linear augmented plane wave (LAPW) basis set was used. Inside MTs the wave functions are expanded in spherical harmonics up to $l_{\max }^{\mathrm{wf}}=10$ and nonspherical contributions to the electron density as well as potential are considered up to $l_{\text {max }}^{\text {pot. }}=4$. The energy cutoff for the plane wave representation in the interstitial is $E_{\max }^{\mathrm{wf}}=19 \mathrm{Ry}$ for the wave functions and $E_{\max }^{\text {pot. }}=196$ Ry for the potential. With these cutoff parameters a convergence of energy differences better than $1 \mathrm{mRy}$ is achieved. Results for the $(\sqrt{2}$

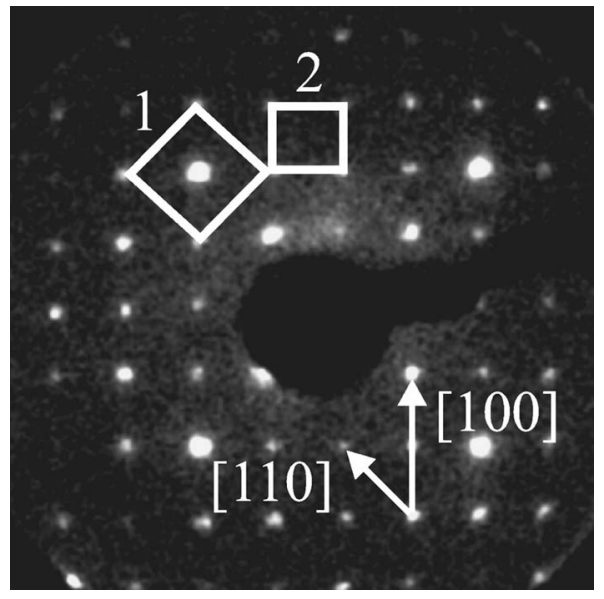

FIG. 3. A $(\sqrt{2} \times \sqrt{2}) R 45^{\circ}$ LEED diffraction pattern of a $\mathrm{Fe}_{3} \mathrm{O}_{4}(100)$ surface. The $p(1 \times 1)$ as well as the $(\sqrt{2} \times \sqrt{2}) R 45^{\circ}$ unit cells are marked by 1 and 2 , respectively. The main crystallographic axes are shown by arrows. The energy of the electron beam is $107 \mathrm{eV}$.

$\times \sqrt{2}) R 45^{\circ}$ unit cell are obtained with four $k_{\|}$points in the irreducible part of the Brillouin zone.

\section{RESULTS AND DISCUSSION}

Figure 3 shows a typical LEED pattern of the (100)oriented surface of an epitaxial $\mathrm{Fe}_{3} \mathrm{O}_{4}$ film grown on $\mathrm{MgO}(100)$ substrate. A clear $(\sqrt{2} \times \sqrt{2}) R 45^{\circ}$ reconstruction with respect to the (100)-unreconstructed $\mathrm{Fe}_{3} \mathrm{O}_{4}$ surface unit cell is visible. A lattice constant of $8.4 \pm 0.15 \AA$ was measured from the observed LEED patterns.

A STM overview of a $(\sqrt{2} \times \sqrt{2}) R 45^{\circ}$ reconstructed $\mathrm{Fe}_{3} \mathrm{O}_{4}(100)$ surface is shown in Fig. 4. Atomically flat terraces with the step edges of nondefined shape can be clearly seen, revealing a layer-by-layer growth. The average step height was found to be about $2.1 \AA$ (see height profile in Fig. 4 ), which corresponds to the distance between adjacent $A-A$ or $B-B$ planes of the bulk $\mathrm{Fe}_{3} \mathrm{O}_{4}$ structure. Atomically resolved rows running along the [011] and [0 $\overline{1} 1]$ directions are clearly visible in this STM image (Fig. 4). The rows on neighboring terraces are oriented in a perpendicular fashion. A change of the row direction can be also observed within a terrace with the transition regions appearing as lines along the [001] or [010] directions (marked by black lines in Fig. $4)$.

In the high-resolution STM image of a typical terrace (Fig. 5), rows consisting of bright protrusions are imaged. The distance between two bright protrusions within the same row was measured to be about $3 \AA$, and the distance between two rows is about $6 \AA$. Alternating pairs of bright protrusions within each row are shifted into the same direction perpendicular to the rows, resulting in a wavelike surface structure. Regularly ordered large dark depressions appear at the wide openings of the waves. The centers of these depressions are separated by $8.4 \AA$ and have a $(\sqrt{2} \times \sqrt{2}) R 45^{\circ}$ symmetry. Similar square-lattice structure types were also observed in STM measurements by Stanka et al. ${ }^{19}$ It should be men- 

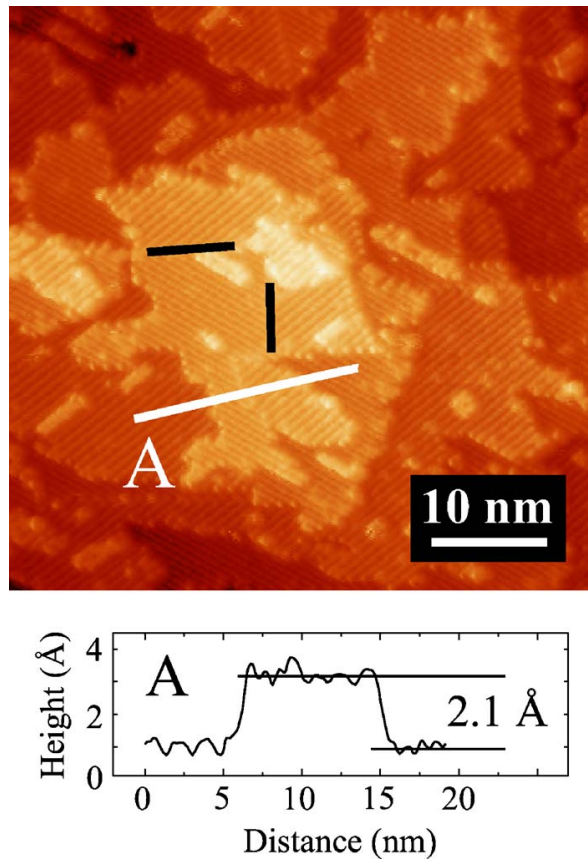

FIG. 4. (Color online) $50 \times 50 \mathrm{~nm}^{2}$ STM image of a $(\sqrt{2}$ $\times \sqrt{2}) R 45^{\circ}$ reconstructed $\mathrm{Fe}_{3} \mathrm{O}_{4}(100)$ surface. Tunneling parameters: $U_{T}=1.1 \mathrm{~V}, I_{T}=1.2 \mathrm{nA}$. A line profile (A) corresponds to the STM height profile along the white line shown in the STM image showing step heights of about $2.1 \AA$.

tioned that careful STM investigation of the $\mathrm{Fe}_{3} \mathrm{O}_{4}(100)$ sample surface performed after the deposition did not reveal any surface reconstructions other than the $(\sqrt{2} \times \sqrt{2}) R 45^{\circ}$.

The interpretation of the constant-current STM images of oxide surfaces is a nontrivial issue due to the strong variations in local density of states (LDOS) on the oxide surface. Band structure calculations by Zhang and Satpathy ${ }^{8}$ for the bulk $\mathrm{Fe}_{3} \mathrm{O}_{4}$ employing LSDA reveal that the $\mathrm{O} 2 p$ orbitals lie well below the Fermi level and the bands near $E_{F}$ are primarily of $\mathrm{Fe} 3 d$ character of $\mathrm{Fe}_{B}$. Thus, it can be concluded that STM images of the $\mathrm{Fe}_{3} \mathrm{O}_{4}(100)$ surface are generally domi-

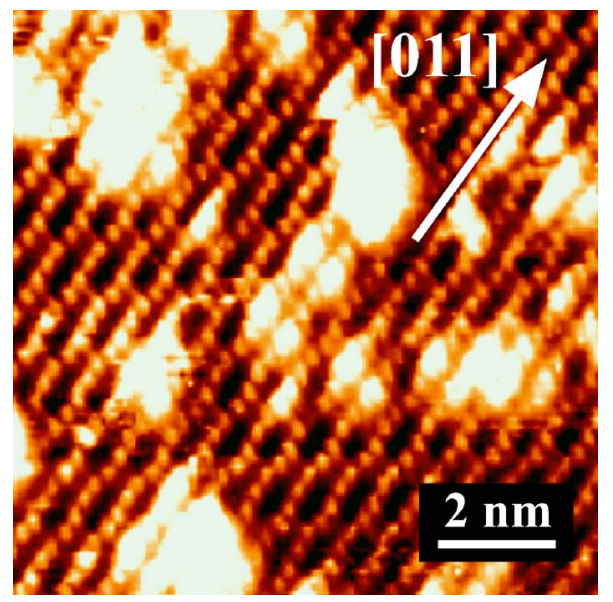

FIG. 5. (Color online) STM image $\left(11 \times 11 \mathrm{~nm}^{2}\right)$ of $\mathrm{Fe}_{3} \mathrm{O}_{4}(100)$ surface showing a wavelike atomic structure which gives rise to a $(\sqrt{2} \times \sqrt{2}) R 45^{\circ}$ periodicity: $\left(U_{T}=1.1 \mathrm{~V}, I_{T}=1.2 \mathrm{nA}\right)$.
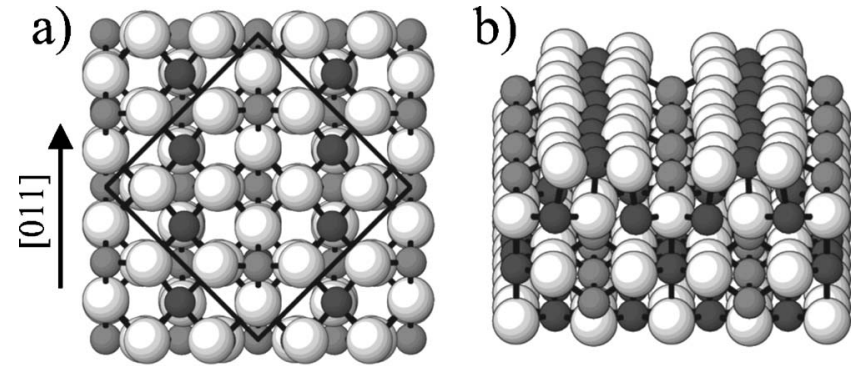

c)

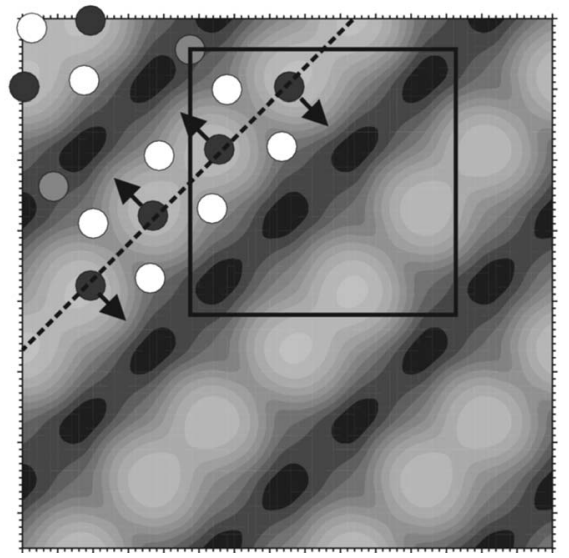

FIG. 6. Top (a) and side (b) view of the modified $B$ termination predicted from the DFT calculations, (c) simulated STM image of the modified $B$ termination. The positions of the surface oxygen, octahedral iron, and subsurface tetrahedral iron are marked by white, gray, and black circles, respectively. The black open square in (a) and (c) denotes the $(\sqrt{2} \times \sqrt{2}) R 45^{\circ}$ unit cell. The dashed line in (c) is a guide for the eyes to emphasize the displacements of surface $\mathrm{Fe}_{B}$ perpendicular to the [011] direction.

nated by iron cations, and that the bright spots within the rows in Fig. 5 are most likely $\mathrm{Fe}_{B}$ ions, which is in agreement with other STM studies. ${ }^{13,15,16,19}$ However, the precise atomic structure of the $\mathrm{Fe}_{3} \mathrm{O}_{4}(100)$ surface remains unclear.

In order to resolve the structure and composition of the surface layer, DFT calculations for a variety of stoichiometric and nonstoichiometric models of $\mathrm{Fe}_{3} \mathrm{O}_{4}$ were performed. In the framework of $a b$ initio atomistic thermodynamics, a surface phase diagram of $\mathrm{Fe}_{3} \mathrm{O}_{4}(100)$ as a function of the oxygen pressure was compiled. ${ }^{24}$ The most striking result is that a modified bulk termination with a $B$ layer showing a wavelike structure has the lowest energy over a broad range of oxygen pressures. Unlike the traditional view of a reconstruction as an ordering of surface defects, in this structure the $(\sqrt{2} \times \sqrt{2}) R 45^{\circ}$ reconstruction emerges as a result of a Jahn-Teller-distortion of the surface layer.

Figure 6 shows the top (a) and the side (b) views of the theoretically predicted energetically most favorable surface structure. A strong lateral relaxation of $0.30 \AA$ of the oxygen atoms with a missing $\mathrm{Fe}_{A}$ neighbor towards the $\mathrm{Fe}_{B}$ row invokes an alternating shift of $\mathrm{Fe}_{B}$ pairs by $\pm 0.09 \AA$ perpendicular to the $\mathrm{Fe}_{B}$ row. Due to these lateral relaxations a wavelike pattern along the [011] direction develops on the surface with neighboring $B$ rows running in antiphase to each other.

To interpret the atomically resolved STM images we performed STM simulations of the modified $B$ termination pre- 
dicted by DFT calculations. The simulated STM image in Fig. 6(c) is obtained in the constant current mode using the Tersoff-Hamann approximation. ${ }^{42}$ The charge density was integrated from $E_{F}$ to $E_{F}+1.1 \mathrm{eV}$, which corresponds to the voltage used in the STM measurements presented above. In Fig. 6(c) light protrusions mark the positions of the surface $\mathrm{Fe}_{B}$. As indicated by the arrows, alternating pairs of such protrusions are shifted perpendicular to the [011] direction giving rise to a wavelike pattern. Although the wavelike structure is clearly visible in the side view of the DFT structure in Fig. 6(b) it is much less pronounced than in the experimentally obtained STM image in Fig. 5. This may be attributed to a softness of the surface with respect to vibrational modes.

Comparison of the measured and calculated work function $(\Phi)$ of the $\mathrm{Fe}_{3} \mathrm{O}_{4}(100)$ surface gives further support for the modified $B$ termination. For this experiment epitaxial $\mathrm{Fe}_{3} \mathrm{O}_{4}(100)$ films were grown on a W(100) single crystal. The work function is determined from the cutoff of the photoelectron spectra. The work-function values of $\mathrm{W}(100)$ $(4.63 \pm 0.15 \mathrm{eV})$ and $\mathrm{Fe}(100)(4.07 \pm 0.15 \mathrm{eV})$ were measured as reference and the latter agrees well the value for an $\mathrm{Fe}(100)$ whisker. ${ }^{43} \mathrm{~A}$ work function of $(5.20 \pm 0.15) \mathrm{eV}$ is measured for the $\mathrm{Fe}_{3} \mathrm{O}_{4}(100)$ surface. The DFT calculations show that the formation of the modified $B$ termination is connected not only with an energy gain of approximately $20 \mathrm{meV} / \AA^{2}$ with respect to the ideal $B$ termination, but also with a reduction of the work function of $0.46 \mathrm{eV}$ from $5.78 \mathrm{eV}$ for the bulk truncated to $5.32 \mathrm{eV}$ for the modified configuration. A reduction of $\Phi$ indicates a decrease of the dipole moment induced on the surface, i.e., a decrease in polarity. We note that the measured $\Phi=5.20 \pm 0.15 \mathrm{eV}$ and the calculated value of the modified $B$ termination $\Phi$ $=5.32 \mathrm{eV}$ are in good agreement, thus giving another proof for the validity of the proposed structural model.

In the bulk, magnetite shows a half-metallic behavior with a band gap in the majority spin channel of approximately $1.5 \mathrm{eV}$ and a $100 \%$ spin-polarization due to the $t_{2 g}$ states of $\mathrm{Fe}_{B}$ at $E_{F}$ in the minority spin channel. ${ }^{8}$ As will be discussed, SP-PES measurements and DFT calculations indicate that the stabilization of the $\mathrm{Fe}_{3} \mathrm{O}_{4}(100)$ surface and the unusual lattice distortions are connected with strong changes in the electronic properties.

Figure 7 shows representative spin-polarized photoemission spectra together with total photoemission intensity of the $\mathrm{Fe}_{3} \mathrm{O}_{4} / \mathrm{MgO}(100)$ system as a function of binding energy. The position of the Fermi energy corresponds to that of a polycrystalline $\mathrm{Au}$ foil in the electrical contact with the sample. The spectra were normalized to the current recorded from the last Au-coated mirror of the beamline. The photon energy was tuned in $2-\mathrm{eV}$ steps over the range $40-60 \mathrm{eV}$, including the $\mathrm{Fe} 3 p-3 d$ excitation threshold at $58 \mathrm{eV}$, where a strong resonance behavior was observed. In the region between $0 \mathrm{eV}$ and $9 \mathrm{eV}$ of binding energy, considerable changes in intensity as well as in spectral shape of the features derived from the $\mathrm{O} 2 p$ and Fe $3 d$ states can be observed as a function of the photon energy. Only weak dispersions (if any) of the photoemission features were observed along the $\Gamma-X$ direction of the $\mathrm{Fe}_{3} \mathrm{O}_{4}$ Brillouin zone. In comparison to

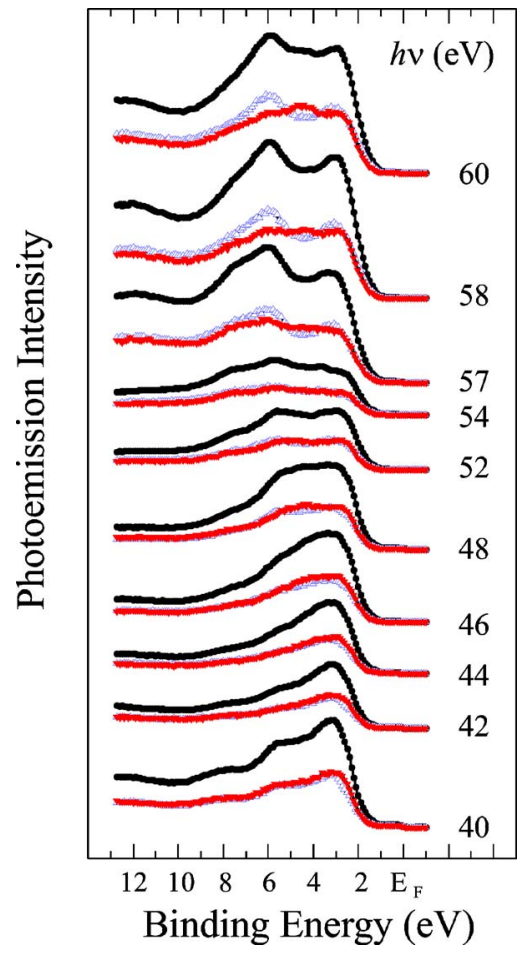

FIG. 7. (Color online) Spin-resolved valence band photoemission spectra (spin up: blue open up triangle; spin down: red solid down triangle) and total intensity (black solid circle) of a $\mathrm{Fe}_{3} \mathrm{O}_{4}(100)$ thin film on $\mathrm{MgO}(100)$ as a function of binding energy recorded at $\mathrm{RT}$ in normal emission at $42-60 \mathrm{eV}$ photon energy.

the present study, well-pronounced band dispersions were reported on the $\mathrm{Fe}_{3} \mathrm{O}_{4}(111)^{30}$ and $\mathrm{Fe}_{3} \mathrm{O}_{4}(100)^{44}$ which can be due to the better angle resolution of $\pm 1^{\circ}$ compared to the acceptance angle of $6^{\circ}$ used in the present study. In the valence band of $\mathrm{Fe}_{3} \mathrm{O}_{4}(100)$ several photoemission features can be clearly observed: a peak at $0.5 \mathrm{eV}$, a small shoulder at $2.2 \mathrm{eV}$, peaks at $3 \mathrm{eV}$ and $5.9 \mathrm{eV}$, and a shoulder at $7.6 \mathrm{eV}$. The positions as well as the general trend of the resonant enhancement of these features is very similar to those already reported for the $\mathrm{Fe}_{3} \mathrm{O}_{4}(111)$ thin films. ${ }^{30}$ However, a more detailed analysis of the origin of the photoemission features and their behavior is beyond the scope of the present paper and will be presented elsewhere. ${ }^{44}$

Figure 8 shows the spin-resolved photoemission spectra together with the total photoemission intensity recorded in normal emission with $h \nu=58 \mathrm{eV}$ (a), the same spectra in the region near $E_{F}(\mathrm{~b})$, and the resulting spin-polarization values (c) as a function of the binding energy relative to the Fermi level of the $\mathrm{Fe}_{3} \mathrm{O}_{4}(100)$ film. The value of $-(55 \pm 10) \%$ at $E_{F}$ measured on the $\mathrm{Fe}_{3} \mathrm{O}_{4}(100)$ surface at RT is significantly lower than the one for the (111) surface $(-80 \%)^{36}$ as well as the $-100 \%$ predicted by LSDA calculations. ${ }^{8}$ However, these band structure calculations were performed for the bulk material not taking into account surface structure effects, which can prevail in the ultraviolet photoemission spectroscopy measurements.

We have now calculated the surface electronic structure of $\mathrm{Fe}_{3} \mathrm{O}_{4}(100)$ terminated by a wavelike $B$ layer and the spinresolved density of states is shown in Fig. 9(b). The most 


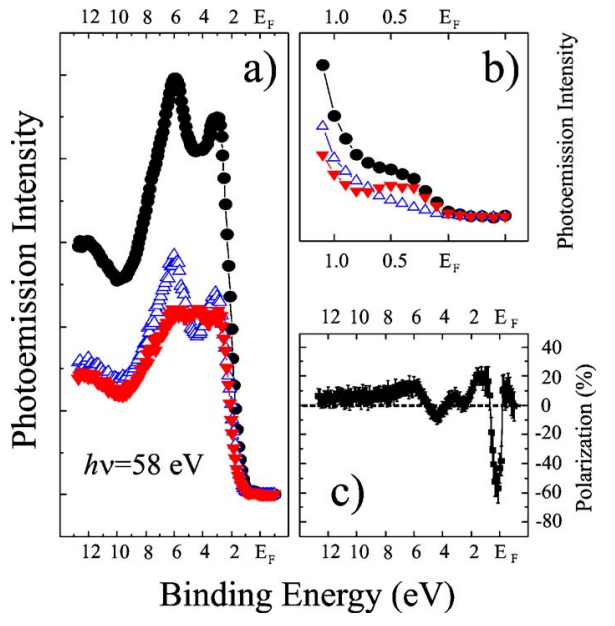

FIG. 8. (Color online) (a) Spin-resolved photoemission spectra (spin up: blue open up triangles; spin down: red solid down triangles; total photoemission intensity: black solid circles) of the $\mathrm{Fe}_{3} \mathrm{O}_{4}(100) / \mathrm{MgO}(100)$ system recorded with $h \nu=58 \mathrm{eV}$ in normal emission, (b) the region near $E_{F}$, and (c) the spin polarization as function of binding energy of the $\mathrm{Fe}_{3} \mathrm{O}_{4}(100) / \mathrm{MgO}(100)$ system.

striking feature of the spin-polarized bands (Fig. 9) are the states that appear in the band gap of the majority subband leading to a reduction of the spin polarization to about $-40 \%$ at $E_{F}$ and a loss of half-metallicity at the $\mathrm{Fe}_{3} \mathrm{O}_{4}(100)$ surface. The calculated spin-polarization value is somewhat lower than $-(55 \pm 10) \%$ obtained from the photoemission measurements. A possible reason for the difference between the calculated and the experimentally measured spin-polarization values may be the finite size of the slab used to model the $\mathrm{Fe}_{3} \mathrm{O}_{4}(100)$. As the states at the Fermi level are relevant for the transport properties of the material their character was analyzed in detail. The spatial distribution of the electronic states in the majority spin channel within the surface plane displayed in Fig. 9(a), shows that these are a hybridization of $d_{x^{2}-y^{2}}$ states of octahedral iron in the surface layer and $p_{x}, p_{y}$ states of the surface oxygen without a subsurface tetrahedral iron neighbor.

In order to gain an understanding of how the electronic structure changes from bulk to the surface as well as to resolve the influence of the surface reconstruction, we have plotted the projected DOS of a $\mathrm{Fe}_{B}$ in the bulk [Fig. 9(c)] as well as on the surface in the ideal [Fig. 9(d)] and modified $B$ termination [Fig. 9(e)]. In the bulk the crystal field splitting of the $e_{g}$ and $t_{2 g}$ states of the octahedrally coordinated iron amounts to $2.0 \mathrm{eV}$. In the majority spin channel both the $e_{g}$ and $t_{2 g}$ are occupied while in the minority spin channel the $e_{g}$ states are unoccuppied and lie $2 \mathrm{eV}$ above the Fermi level and $E_{F}$ crosses the $t_{2 g}$ band. On the surface, due to the broken symmetry in the $z$ direction, the degeneracy within the $e_{g}$ doublet and $t_{2 g}$ triplet is lifted. In the majority spin channel $d_{x^{2}-y^{2}}\left(d_{z^{2}}\right)$ states are shifted to higher (lower) energies and the former cross the Fermi level even in the ideal (unrelaxed) $B$ termination [Fig. 9(d)].

The DFT results indicate that the origin of the wavelike $(\sqrt{2} \times \sqrt{2}) R 45^{\circ}$ pattern observed in STM is a Jahn-Teller distortion. The main feature associated with the distortion of the

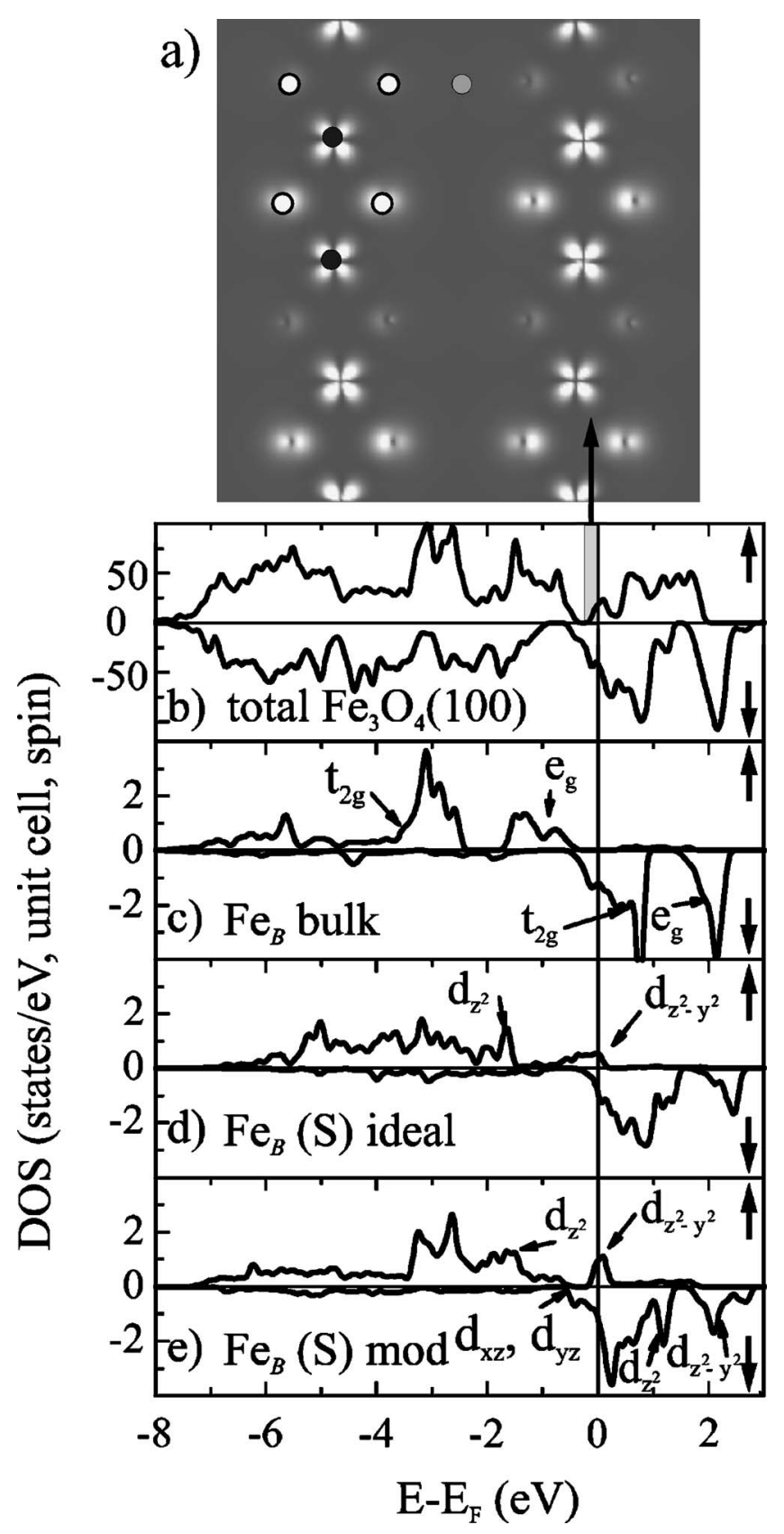

FIG. 9. (a) Charge density distribution of the surface states in the majority spin channel from $-0.2 \mathrm{eV}$ to $E_{F}$ in the surface layer. The positions of the surface oxygen, octahedral iron, and subsurface tetrahedral iron are marked by white, gray, and black circles, respectively. Spin-resolved density of states of $\mathrm{Fe}_{3} \mathrm{O}_{4}(100)$ surface terminated by a modified $B$ layer: $^{24}$ (b) total DOS; projected DOS of $\mathrm{Fe}_{B}$ in the bulk (c) as well as on the surface in the ideal (d) and modified (e) $B$ layer.

surface atoms is the opening of a band gap in the majority spin channel between $-0.4 \mathrm{eV}$ and $-0.1 \mathrm{eV}$ [cf. Fig. 9(e)]. This leads to a shift through the Fermi level and partial deoccupation of $d_{x^{2}-y^{2}}$ states of the surface octahedral iron. The depletion of states in the majority spin channel is accompanied by occupation of states in the minority spin channel. This effect, referred to as spin flip, results in a substantial reduction of the magnetic moments of the surface $\mathrm{Fe}_{B}$ from 
$3.6 \mu_{\mathrm{B}}$ for the $B$ termination with bulk positions of the atoms to $3 \mu_{\mathrm{B}}$ for the modified $B$ termination.

As a result of the spin flip effect in the minority spin channel, $d_{x z}$ and $d_{y z}$ states are partially occupied and shifted to lower energies, while $d_{x y}$ states of the surface iron remain unoccupied at $0.3 \mathrm{eV}$ above $E_{F}$. The $d_{z^{2}}$ states are pulled to lower energies at $1.1 \mathrm{eV}$ above the Fermi level, almost touching the $t_{2 g}$ band and the splitting between $d_{z^{2}}$ and $d_{x^{2}-y^{2}}$ states amounts to $1 \mathrm{eV}$. We note that the positions of the $d$ orbitals differ from the qualitative picture proposed by Shvets et al. ${ }^{21}$ which suggests a small and large splitting of the orbitals of $\mathrm{Fe}^{2+}$ and $\mathrm{Fe}^{3+}$, respectively. The authors of Ref. 21 conclude that only $\mathrm{Fe}^{2+}$ may be subject to a Jahn-Teller distortion. The model of Shvets et al. ${ }^{21}$ considers the extreme case where the sixth electron is completely localized at $\mathrm{Fe}^{2+}$, while the DFT-GGA calculation renders an average occupation of $5.38 d$ electrons within the muffin tin for both $\mathrm{Fe}_{B}$ ions in the surface. Recently, Wright et al. ${ }^{45}$ obtained evidence from $x$-ray and neutron powder diffraction that the charge disproportionation between octahedral $B$ iron below the Verwey temperature is 2.4 and 2.6 , which is only $20 \%$ of the ideal value for $\mathrm{Fe}^{2+}$ and $\mathrm{Fe}^{3+}$. This suggests that assigning integer charges to the iron ions may not be appropriate. Still, the influence of strong electron correlation effects on the surface electronic structure remains to be investigated. ${ }^{46}$

\section{CONCLUSION}

The formation of the wavelike pattern along the [011] direction observed by STM is explained using DFT calculations as a Jahn-Teller distortion in the surface $B$ layer. ${ }^{24}$ STM simulations indicate that the contrast in the STM images arises from octahedral iron ions shifted pairwise perpendicular to the [011] direction. The work-function value of the $\mathrm{Fe}_{3} \mathrm{O}_{4}(100)$ surface extracted from the cutoff of the photoelectron spectra are consistent with those predicted on the basis of DFT calculations. Both the spin-resolved photoemission experiments and the DFT-GGA density of states give evidence for a half-metal to metal transition at the $\mathrm{Fe}_{3} \mathrm{O}_{4}(100)$ surface: the measured negative spin polarization of about $-(55 \pm 10) \%$ at the Fermi level and the theoretical value of $-40 \%$ are significantly lower than the $-100 \%$ predicted by LSDA calculations for the bulk magnetite crystal as well as the $-(80 \pm 5) \%$ obtained for the $\mathrm{Fe}_{3} \mathrm{O}_{4}(111)$ films. The electronic states in the majority spin-channel responsible for the reduction of spin polarization are dominated by $d_{x^{2}-y^{2}}$ states of surface $\mathrm{Fe}_{B}$.

\section{ACKNOWLEDGMENTS}

R.P. thanks W. Moritz and M. Todorova for helpful discussions and acknowledges support by the DFG, PE 883 . Part of this work was supported by SFB 513.
*Electronic address: mikhail.fonin@uni-konstanz.de

${ }^{1}$ N. Tsuda, K. Nasu, A. Yanase, and K. Siratori, Electronic Conduction in Oxides (Springer, Berlin, 1991).

${ }^{2}$ Spectroscopy of Mott Insulators and Correlated Metals, edited by A. Fujimori and Y. Tokura (Springer, Berlin, 1995).

${ }^{3}$ J. G. Bednorz and K. A. Müller, Z. Phys. B: Condens. Matter 64, 189 (1986); J. G. Bednorz and K. A. Müller, Rev. Mod. Phys. 60, 585 (1988).

${ }^{4}$ R. von Helmolt, J. Wecker, B. Holzapfel, L. Schultz, and K. Samwer, Phys. Rev. Lett. 71, 2331 (1993).

${ }^{5}$ A. P. Ramirez, J. Phys.: Condens. Matter 9, 8171 (1997).

${ }^{6}$ J. M. D. Coey, M. Viret, and S. von Molnar, Adv. Phys. 48, 167 (1999).

${ }^{7}$ A. Yanase and K. Siratori, J. Phys. Soc. Jpn. 53, 312 (1984).

${ }^{8}$ Z. Zhang and S. Satpathy, Phys. Rev. B 44, 13319 (1991).

${ }^{9}$ P. Seneor, A. Fert, J.-L. Maurice, F. Montaigne, F. Petroff, and A. Vaurès, Appl. Phys. Lett. 74, 4017 (1999).

${ }^{10}$ H. Matsuda, M. Takeuchi, H. Adachi, M. Hiramoto, N. Matsukawa, A. Odagawa, K. Setsune, and H. Sakakima, Jpn. J. Appl. Phys., Part 2 41, L387 (2002).

${ }^{11}$ G. Hu and Y. Suzuki, Phys. Rev. Lett. 89, 276601 (2002).

${ }^{12}$ P. W. Tasker, J. Phys. C 12, 4977 (1979); P. W. Tasker, Philos. Mag. A 12, 4977 (1979).

${ }^{13}$ G. Tarrach, D. Bürgler, T. Schaub, R. Wiesendanger, and H. Güntherodt, Surf. Sci. 285, 1 (1993).

${ }^{14}$ Y. J. Kim, Y. Gao, and S. A. Chambers, Surf. Sci. 371, 358 (1997).

${ }^{15}$ J. M. Gaines, P. J. H. Bloemen, J. T. Kohlhepp, C. W. T. BulleLieuwma, R. M. Wolf, R. M. Reinders, R. M. Jungblut, P. A. A. van der Heijden, J. T. W. M. van Eemeren, J. aan de Stegge, and W. J. M. de Jonge, Surf. Sci. 373, 85 (1997).

${ }^{16}$ J. F. Anderson, M. Kuhn, U. Diebold, K. Shaw, P. Stoyanov, and D. Lind, Phys. Rev. B 56, 9902 (1997).

${ }^{17}$ F. C. Voogt, T. Fujii, P. J. M. Smulders, L. Niesen, M. A. James, and T. Hibma, Phys. Rev. B 60, 11193 (1999).

${ }^{18}$ S. A. Chambers, S. Thevuthasan, and S. A. Joyce, Surf. Sci. 450, L273 (2000).

${ }^{19}$ B. Stanka, W. Hebenstreit, U. Diebold, and S. A. Chambers, Surf. Sci. 448, 49 (2000).

${ }^{20}$ G. Mariotto, S. Murphy, and I. V. Shvets, Phys. Rev. B 66, 245426 (2002).

${ }^{21}$ I. V. Shvets, G. Mariotto, K. Jordan, N. Berdunov, R. Kantor, and S. Murphy, Phys. Rev. B 70, 155406 (2004).

${ }^{22}$ J. R. Rustad, E. Wasserman, and A. R. Felmy, Surf. Sci. 432, L583 (1999).

${ }^{23}$ J. P. LaFemina, Crit. Rev. Surf. Chem. 3, 297 (1994).

${ }^{24}$ R. Pentcheva, F. Wendler, H. L. Meyerheim, W. Moritz, N. Jedrecy, and M. Scheffler, Phys. Rev. Lett. 94, 126101 (2005).

${ }^{25}$ W. Moritz, R. Pentcheva, S. Frank, and D. Schrupp (unpublished).

${ }^{26}$ A. V. Mijiritskii and D. O. Boerma, Surf. Sci. 486, 73 (2001).

${ }^{27}$ R. Wiesendanger, I. V. Shvets, D. Bürgler, G. Tarrach, H. J. Güntherodt, J. M. D. Coey, and S. Gräser, Science 255, 583 (1992).

${ }^{28}$ F. M. F. de Groot, J. C. Fuggle, B. T. Thole, and G. A. Sawatzky, Phys. Rev. B 42, 5459 (1990).

${ }^{29}$ H.-J. Kim, J.-H. Park, and E. Vescovo, Phys. Rev. B 61, 15284 (2000). 
${ }^{30}$ Y. Q. Cai, M. Ritter, W. Weiss, and A. M. Bradshaw, Phys. Rev. B 58, 5043 (1998).

${ }^{31}$ H.-J. Kim, J.-H. Park, and E. Vescovo, Phys. Rev. B 61, 15288 (2000).

${ }^{32}$ Yu. S. Dedkov, M. Fonin, D. V. Vyalikh, J. O. Hauch, S. L. Molodtsov, U. Rüdiger, and G. Güntherodt, Phys. Rev. B 70, 073405 (2004).

${ }^{33}$ S. F. Alvarado, W. Eib, F. Meier, D. T. Pierce, K. Sattler, H. C. Siegmann, and J. P. Remeika, Phys. Rev. Lett. 34, 319 (1975).

${ }^{34}$ S. F. Alvarado, M. Erbudak, and P. Munz, Phys. Rev. B 14, 2740 (1976).

${ }^{35}$ S. F. Alvarado and P. S. Bagus, Phys. Lett. 67A, 397 (1978).

${ }^{36}$ Yu. S. Dedkov, U. Rüdiger, and G. Güntherodt, Phys. Rev. B 65, 064417 (2002).

${ }^{37}$ M. Fonin, Yu. S. Dedkov, J. Mayer, U. Rüdiger, and G. Güntherodt, Phys. Rev. B 68, 045414 (2003).

${ }^{38}$ S. A. Morton, G. D. Waddill, S. Kim, Ivan K. Schuller, S. A. Chambers, and J. G. Tobin, Surf. Sci. 513, L451 (2002).
${ }^{39}$ D. J. Huang, C. F. Chang, J. Chen, L. H. Tjeng, A. D. Rata, W. P. Wu, S. C. Chung, H. J. Lin, T. Hibma, and C. T. Chen, J. Magn. Magn. Mater. 239, 261 (2002).

${ }^{40}$ P. Blaha, K. Schwarz, G. K. H. Madsen, D. Kvasnicka, and J. Luitz, WIEN2K, An Augmented Plane Wave+Local Orbitals Program for Calculating Crystal Properties (Karlheinz Schwarz, Techn. Univ. Wien, Austria), 2001. ISBN 3-9501031-1-2.

${ }^{41}$ J. P. Perdew, K. Burke, and M. Ernzerhof, Phys. Rev. Lett. 77, 3865 (1996).

${ }^{42}$ J. Tersoff and D. R. Hamann, Phys. Rev. B 31, 805 (1985).

${ }^{43}$ J. J. Paggel, C. M. Wei, M. Y. Chou, D.-A. Luh, T. Miller, and T.-C. Chiang, Phys. Rev. B 66, 233403 (2002).

${ }^{44}$ Yu. S. Dedkov, M. Fonin, U. Rüdiger, and G. Güntherodt (unpublished).

${ }^{45}$ J. P. Wright, J. P. Attfield, and P. G. Radaelli, Phys. Rev. Lett. 87, 266401 (2001)

${ }^{46}$ R. Pentcheva (unpublished). 